\title{
Update in diagnostic imaging of the thymus and anterior mediastinal masses
}

\author{
Francesco Gentili ${ }^{1}$, Veronica Pelini ${ }^{1}$, Gabriele Lucii ${ }^{1}$, Luca Luzzi ${ }^{2}$, Francesco Giuseppe Mazzei ${ }^{3}$, \\ Alfonso Fausto ${ }^{3}$, Luca Volterrani ${ }^{1}$, Maria Antonietta Mazzei ${ }^{1}$
}

${ }^{1}$ Department of Medicine, Surgery and Neuro Sciences, Diagnostic Imaging, University of Siena, Azienda Ospedaliera Universitaria Senese, Siena, Italy; ${ }^{2}$ Thoracic Surgery Unit, Department of Medicine, Surgery and Neuro Sciences, Diagnostic Imaging, University of Siena, Azienda Ospedaliera Universitaria Senese, Siena, Italy; ${ }^{3}$ Diagnostic Imaging, University of Siena, Azienda Ospedaliera Universitaria Senese, Siena, Italy

Contributions: (I) Conception and design: Gentili F, Mazzei MA; (II) Administrative support: Fausto A, Volterrani L; (III) Provision of study materials or patients: Gentili F, Lucii G, Luzzi L, Mazzei FG; (IV) Collection and assembly of data: Gentili F, Pelini V, Mazzei MA; (V) Data analysis and interpretation: Gentili F, Pelini V, Lucii G, Mazzei MA; (VI) Manuscript writing: All authors; (VII) Final approval of manuscript: All authors.

Correspondence to: Francesco Gentili, MD. Department of Medicine, Surgical and Neuro Sciences, Diagnostic Imaging, University of Siena, Azienda Ospedaliera Universitaria Senese, Viale Bracci 10, 53100 Siena, Italy. Email: francescogentili@gmail.com.

\begin{abstract}
Anterior mediastinal masses include a wide spectrum of malignant and benign pathologies with a large percentage represented by thymic lesions. Distinguishing these masses on diagnostic imaging is fundamental to guide the proper management for each patient. This review illustrates possibilities and limits of different imaging modalities to diagnose a lesion of the anterior mediastinum with particular attention to thymic disease.
\end{abstract}

Keywords: Thymoma; anterior mediastinal neoplasm; imaging modalities

Submitted Jan 20, 2019. Accepted for publication May 09, 2019.

doi: $10.21037 /$ gs.2019.05.06

View this article at: http://dx.doi.org/10.21037/gs.2019.05.06

\section{Introduction}

Thymus is a lymphoid organ of the immune system, the site where the haemopoietic precursor cells evolve into T-cells, which are fundamental for the adaptive immune system. It emerges from the $3^{\text {rd }}$ and $4^{\text {th }}$ branchial sack, containing elements from all three germinal films, even if the main cell populations are the thymocytes and the reticular epithelial cells. Its development begins in the $6^{\text {th }}$ gestational week and from the $8^{\text {th }}$ gestational week it starts migrating reaching its final position in the anterior mediastinum after the fusion of its two lobes. During its development, until the $9^{\text {th }}$ gestational week, thymus remains epithelial; by the $10^{\text {th }}$ week, small lymphoid cells migrate from fetal liver and bone marrow, leading to lobulation of the gland. Thymus is protected by a connective tissue capsule, the septa of which penetrate into the tissue and divides it into incomplete lobules. Each lobule has a peripheral dark zone called cortex and a middle lighter zone called medulla; the latter differentiation is completed by 14 and 16 weeks (1). The capsule is made up of inner and outer layers of collagen and reticular fibers. It continues to become larger, reaching its maximum absolute weight at puberty; during adult age it undergoes an involution process during which the epithelial cells are replaced from adipose tissue and thus it is difficult to recognize on imaging studies performed in healthy adults (1). However, once the thymus becomes pathological, the gland demonstrates a variety of clinical and radiological manifestations that require comprehensive understanding of each entity. Thus, it is really important to recognize normal thymus and distinguishing it from its pathological manifestations which could be benign (thymic cists, thymic hyperplasia) or malignant (thymic neoplasm). Since the thymus is anatomically located in the anterior superior mediastinum, where the $50 \%$ of all mediastinal masses could be located, it plays the main role in the differential diagnosis of anterior mediastinal masses $(2,3)$. Moreover, 
because thymus migrates from the $3^{\text {rd }}$ and $4^{\text {th }}$ branchial pouches to the anterior mediastinum, ectopic thymus tissue or ectopic thymoma can occur anywhere long this pathway, often manifesting as a neck mass. Thus, to be awareness of the embryologic features of the thymus is important in recognizing these entities (1).

The aim of this article is to provide a comprehensive review on thymic lesions and their differential diagnosis, throughout imaging.

\section{The mediastinum and the "appearance" of normal thymus in adults}

The mediastinum is an anatomic space in the chest located between the two pleural spaces, behind the posterior sternal table, extending superiorly to the thoracic inlet, posteriorly to the anterior longitudinal ligament of the spine and paraspinal sulci, and inferiorly to the diaphragm. It contains vital structures such as hearth, great vessels, trachea and main bronchi, esophagus, thymus, venous and lymphatic structures and nerve tissue; in clinical and radiological practice it is frequent to find masses into this space, especially in the anterior compartment $(50 \%$ of all mediastinal masses) and the goal of radiology is to define their specific origin in order to guide clinicians and surgeons to the best clinical or surgical treatment. Several divisions in different compartments have been proposed over the years; the most famous is the Felson's division that divides the mediastinum into three compartments (anterior, middle and posterior compartment) on lateral chest radiography $(4,5)$.

The main problem of this classification is that it is based on the lateral chest radiography. Felson, although he never classified the mediastinum, proposed a way to identify a mass based on its position with respect to two lines drawn. The dividing line between the anterior and middle mediastinum is detected along the anterior portion of the cardiac posterior margin. Within the center of the body (i.e., the retrosternal space), the sagittal plane of the section shows the anterior mediastinal area before the anterior tracheal wall, the large vessels and the pericardium. Nonetheless, on the left side of the mediastinum, the posterior limit of the anterior mediastinum diffuses deeply along the aortic arch and the left bone, and this anatomical complication produces incoherence. That is to say that the anterior compartment could include some masses both in the anterior mediastinum and in the middle mean, on a lateral chest radiograph (6).
Furthermore, due to the fact that it is reasonably common in radiological practice to find a mediastinal mass as an occasional finding during a computed tomography (CT) scan for other reasons and since moreover the therapeutic and eventually surgical strategy is established by the clear definition of the site of the mass and of its connection with the other neighbouring structures, only possible with CT or magnetic resonance imaging (MRI), the International Thymus Malignancy Interest Group (ITMIG) proposed a new classification of mediastinal compartments in CT in order to overcome this problem.

In this classification the mediastinum has been divided into prevascular, visceral, and paravertebral compartments corresponding to the previously defined anterior, middle and posterior mediastinal regions (7). The superior and inferior borders are the same for the three compartments and consist of the thoracic inlet and diaphragm, respectively. The posterior border of the prevascular compartment is the anterior part of the pericardium. That means that this region wraps around the heart up to the right and left pulmonary hila, whereas the posterior line of the visceral compartment consists of a curved vertical line drawn $1 \mathrm{~cm}$ posterior to the anterior aspect of the thoracic vertebrae, which is best visualized on a sagittal view. In this way the descending aorta belongs to the visceral compartment $(8,9)$.

Another recent mediastinal classification has been proposed by the Japanese Association for Research on the thymus (JART) (6). This classification recognizes four mediastinal partitions based on oblique CT images: superior part of mediastinum, anterior mediastinum, middle mediastinum and posterior mediastinum, and it is pondered for the differential diagnosis of mediastinal tumors. Recognizing the superior part of the mediastinum has the advantage of making it easy to differentiate an intrathoracic struma or neurogenic mass of the thoracic inlet from other mediastinal masses. We described the limit at the caudate edge of the brachiocephalic vein with the trachea (tracheal midplane). This limit is confidential to thoracic radiologists, physicians and surgeons, because corresponds to an imaginary line of $2 \mathrm{R}$ (right upper paratracheal) and 4R (right lower paratracheal) mediastinal lymph nodes on the International Association for the Study of Lung Cancer (IASLC) lymph node map (10-12). Applying this stratification in the investigation of 445 pathologically demonstrated mediastinal mass, and categorized them into the proposed four compartments by consensus reading, the majority of intrathoracic struma and one-fifth of neurogenic 
Table 1 Definition of the JART mediastinum compartments

\begin{tabular}{|c|c|}
\hline Mediastinal compartment & Definition \\
\hline Prevascular zone & $\begin{array}{l}\text { The anatomical limits of this compartment are the following: superior, the inferior boundary of the superior } \\
\text { portion; inferior, the diaphragm; anterior, the sternum; lateral, the parietal (mediastinal) pleural reflection } \\
\text { (including the lateral rim of the bilateral internal thoracic arteries and veins, and superior and inferior } \\
\text { pulmonary veins); and posterior, the pericardium (including a horizontal line at the posterior rime of the } \\
\text { heart), anterior rims of the left brachiocephalic vein, superior vena cava, superior and inferior pulmonary } \\
\text { veins, ascending aorta, and lateral rim of the aortic arch }\end{array}$ \\
\hline Paravertebral zone & $\begin{array}{l}\text { This compartment is anatomically bounded as follows: superior, the boundary of the superior portion of the } \\
\text { mediastinum; inferior the diaphragm; anterior, the boundary of the middle mediastinum; and } \\
\text { posterior-lateral, a vertical line against the posterior rim of the chest wall at the lateral rim of the lateral } \\
\text { process of the thoracic spine }\end{array}$ \\
\hline
\end{tabular}

JART, Japanese Association for Research on the thymus; IASLC, International Association for the Study of Lung Cancer.

tumors were found in the superior part of the mediastinum. Avoiding this classification would affect differential diagnosis, in that many intrathoracic struma would be categorized as being in the anterior or middle mediastinum.

Differently from formerly described methods of mediastinal subdivision where the heart and great vessels, trachea, and main bronchi are located in the middle mediastinum, whilst the esophagus is located in the posterior mediastinum $(5,13)$ the JART classification did not include the cardiovascular system in the mediastinal compartmentalization (6). The anterior edge of great vessels, pericardium and heart are considered the posterior limit of the anterior mediastinal compartment, whilst the posterior edge of the great vessels, pericardium and heart are the anterior limit of the middle mediastinal compartment. The authors justify this choice stating that esophagus, trachea, and bronchi share an embryological genesis (foregut, endoderm), these should all be classified into the same compartment (the middle mediastinum). Most of the lesions located in the middle mediastinum included foregut cysts, tracheal and esophageal tumors, and lymphadenopathy (Table 1).
From a radiological point of view what constitutes the normal anatomy of thymus is another important issue. After reaching its greatest dimension in relation to body weight before birth, the thymus continues to grow, reaching its maximum weight in puberty; after that it subsequently undergoes involution. This activity starts at puberty. In the course of involution, the epithelial component encounter atrophy, evolving in separated small lymphocytes in profuse adipose tissue. At CT examination thymus become visible as a bilobated triangular structure detected in the anterior mediastinum, most commonly before to the proximal ascending aorta, the pulmonary outflow tract, and the distal superior vena cava until it enters the right atrium. Differentiating thymus from other mediastinal structures may be challenging. The size of a normal thymus has been largely studied with CT and MRI, ranging from $1.1 \mathrm{~cm}$ $( \pm 0.4 \mathrm{~cm})$ to $0.5 \mathrm{~cm}( \pm 0.27 \mathrm{~cm})$, according to the age of the patient at CT examination, and to 1.5 to $2.0 \mathrm{~cm}$ at magnetic resonance $(\mathrm{MR})$ examination. Its thickness appears greater on MRI because of the better identification of thymic tissue partially replaced by fat and the margins of thymus with this modality (1). 

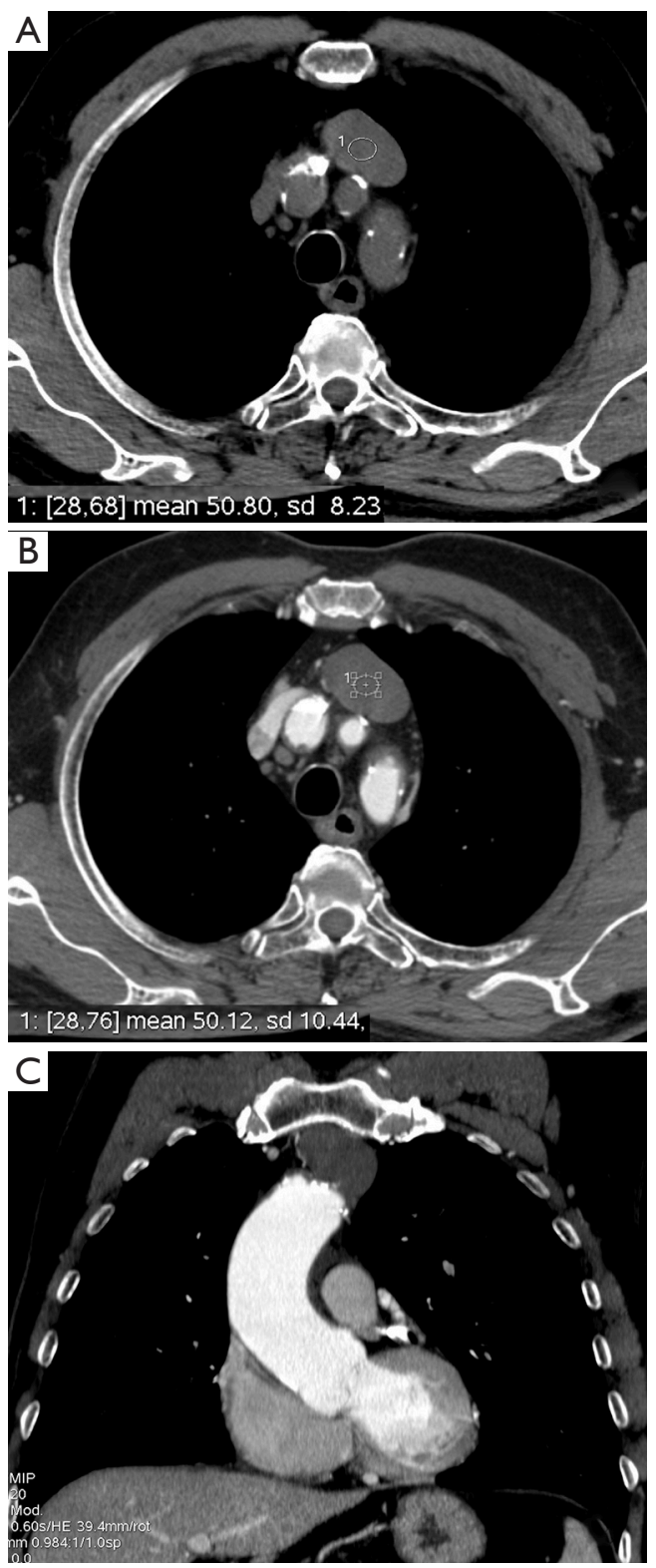

Figure 1 Axial and coronal pre and post-contrast CT images show a thymic cyst in an asymptomatic 73-year-old man who underwent CT scan for studying an aneurysm of abdominal aorta. Comparing pre (A) and post contrast images (B,C), it is clear the absence of contrast uptake by the lesion. CT, computed tomography.

\section{Thymic pathologies}

\section{Benign thymic lesions}

\section{Thymic cysts}

Thymic cysts are $1-3 \%$ of all mediastinal masses; they could be congenital or acquired (Figure 1). Congenital cysts are generally simple, unilocular with thin wall. Acquired cysts have been associated with myasthenia gravis, systemic lupus erythematosus, Sjogren syndrome, aplastic anaemia, radiation therapy and lymphomas; they are generally multilocular with a thick wall and could have signs of inflammation and tendency to recur after resection more frequent than the congenital cysts; furthermore, they could be associated with thymic malignancy (3).

\section{Thymic hyperplasia}

Thymic hyperplasia is defined as a symmetric gland's volume increase; there are two histologic sub types of thymic hyperplasia which are associated with different medical conditions (3). The true thymic hyperplasia occurs when there is a symmetric volume enlargement of the thymus with normal histology. It is commonly due to a rebound from recent stress and occurs after certain conditions such as irradiation or chemotherapy for neoplasms, steroid therapy, pneumonia, surgery or burns; sometimes the gland remains bigger than normal.

The second one is the lymphofollicular hyperplasia, which is a symmetric enlargement due to an increased number of lymphoid follicles in the medulla of the gland; occurring in more than $50 \%$ patients with myasthenia gravis could be also associated with other autoimmune diseases such as systemic lupus erythematosus, rheumatoid arthritis, scleroderma, vasculitis, thyrotoxicosis and Graves' disease $(14,15)$.

\section{Thymic neoplasms}

Thymic neoplasms are divided into epithelial tumors, which are exemplified by thymoma and thymic carcinoma, and nonepithelial tumors which are represented by lymphoma of the thymus, thymic carcinoid, germ cells tumors of the thymus and thymolipoma (3).

\section{Epithelial tumors}

Thymic epithelial tumors are malignant masses emerging from the thymus that take part for $0.2 \%$ to $1.5 \%$ of all malignancies but are the most common non-lymphomatous primary neoplasms of the anterior mediastinum $(8,16)$.

Thymoma is the most common thymic epithelial tumour and it accounts for $15-21.7 \%$ of tumors in the mediastinum and $47 \%$ of tumors in the anterior mediastinum (17). Thymomas are typically slow-growing masses but can show aggressive behavior such as invasion of adjacent thoracic 
Table 2 Histologic classification of thymic tumors according to the $4^{\text {th }}$ edition WHO

\begin{tabular}{ll}
\hline Classification & Definition \\
\hline Thymomas type AB & $\begin{array}{l}\text { Occurrence of bland spindle shaped epithelial cells with lymphocyte-dense areas or with }>10 \% \text { tumor area with a } \\
\text { moderate infiltrate of T-cells }\end{array}$ \\
Thymomas type B1 & $\begin{array}{l}\text { Thymus-like architecture and cytology: abundance of immature T-cells and absence of epithelial cell clusters; } \\
\text { Hassal's corpuscles occur as optional feature }\end{array}$ \\
Thymomas type B2 & $\begin{array}{l}\text { Higher than normal number of polygonal (non-spindle) neoplastic epithelial cells that commonly occur in cluster; } \\
\text { medullary islands optionally occur }\end{array}$ \\
Thymomas type B3 & $\begin{array}{l}\text { Lymphocyte-poor, epithelial-rich tumor which can be differentiated from the type B2 on the visual impression on H/E } \\
\text { staining (B2 looks blue, B3 looks pink) }\end{array}$
\end{tabular}

WHO, World Health Organization.

structures and involvement of the pleura and pericardium; however, long-distance metastatic disease is rare (18).

Thymic carcinoma accounts for approximately $20 \%$ of thymic epithelial neoplasms and the mean age of presentation is 50 years. Aggressive features such as local invasion and intrathoracic lymphadenopathy are much more common in thymic carcinoma than thymoma, and approximately $50 \%$ to $65 \%$ of patients present with distant metastases at the time of diagnosis.

The histologic classification of thymic epithelial tumors has changed during the years as well as their staging. In 2015 the World Health Organization (WHO) published the $4^{\text {th }}$ edition of the classification of thymic tumors (Table 2). This classification system maintained the established nomenclature, in which thymomas are separated from thymic carcinoma and thymomas are subdivided into A, $\mathrm{AB}, \mathrm{B} 1, \mathrm{~B} 2$ and $\mathrm{B} 3$ types based upon the morphology of epithelial tumor cells (polygonal or spindle cells), the relative proportion of the nontumoral lymphocytic component (decreasing from type B1 to B3), and resemblance to normal thymic architecture (19).

In addition, this new classification defines an "atypical type A thymoma variant" for thymoma type A that shows hypercellularity, increased mitotic activity and necrosis and Micronodular thymoma with lymphoid stroma and metaplastic thymoma. The thymic carcinoma was defined in its various histological subtypes: squamous cell (36\%), lymphoepithelioma-like (32\%), undifferentiated (11\%), small cell (8\%), basaloid (4\%), sarcomatoid (4\%), clear cell (2\%), mucoepidermoid (2\%) and adenocarcinoma (1\%). A neuroendocrine differentiation is also recognized (20).

The $4^{\text {th }}$ classification of thymic tumors also introduced the term "combined thymic carcinoma" for tumors that are either composed of different types of thymic carcinoma or for tumors composed by carcinoma and noncarcinoma component (thymoma or carcinoid). If the carcinoma component is very small the tumor cannot be counted in the combined thymic carcinoma but it must be considered in the category of the most represented component. The WHO classification scheme is based on histologic features; however, it has been shown to correlate with the invasiveness and clinical behavior of tumors and with prognosis. In 2017 the $8^{\text {th }}$ edition of TNM/ AJCC classification established for the first time a stage classification of thymic neoplasms including thymoma, thymic carcinoma, thymic neuroendocrine tumors and combined thymic carcinoma (Tables 3-6) (21). In a recent retrospective study Ried et al. conclude that the new TNMstaging system is clinically useful and applicable and it can be used for indication, stage-adapted therapy and prediction of prognosis for overall and recurrence-free survival (22). This classification is determined primarily by levels of local invasion of a thymic malignancy into the surrounding mediastinal structures (T); T1: tumor encapsulated or extending into the mediastinal fat (T1a); may involve the mediastinal pleura (T1b); T2: tumor with direct invasion of the pericardium (either partial or full thickness); T3: tumor with direct invasion into any of the following: lung, brachiocephalic vein, superior vena cava, phrenic nerve, chest wall extra-pericardial pulmonary artery or veins; T4: tumor with invasion into any of aorta, arch vessels, intra-pericardial pulmonary artery, myocardium, trachea or esophagus. The new TNM classification defines nodal involvement $(\mathrm{N})$ by classifying either superficial (N1: anterior mediastinum and lower region) or deep (N2: deep cervical and middle mediastinal nodes) region. Metastatic spread involves most frequently pleura or pericardium (M1a) or less commonly distant, extra-thoracic site (M1b). 
Table 3 Definition of primary thymic tumor

\begin{tabular}{ll}
\hline T category & T description \\
\hline Tx & Primary tumor cannot be assessed \\
T0 & No evidence of primary tumor \\
T1 & Tumor encapsulated or extending into the mediastinal fat; may involve the mediastinal pleura \\
T1a & Tumor with no mediastinal pleura involvement \\
T1b & Tumor with direct invasion of mediastinal pleura \\
T2 & Tumor with direct invasion of the pericardium (either partial or full thickness) \\
T3 & Tumor with direct invasion into any of the following: lung, brachiocephalic vein, superior vena cava, phrenic nerve, chest \\
& wall, or extrapericardial pulmonary artery or veins \\
T4 & Tumor with invasion into any of the following: aorta (ascending, arch, or descending), arch vessels, intrapericardial \\
& pulmonary artery, myocardium, trachea, esophagus
\end{tabular}

T, tumor.

Table 4 Definition of regional lymph node

\begin{tabular}{ll}
\hline N category & N description \\
\hline Nx & Regional lymph nodes cannot be assessed \\
No & No regional lymph node metastasis \\
N1 & Metastasis in anterior (perithymic) lymph nodes \\
N2 & Metastasis in deep intrathoracic or cervical lymph \\
& nodes \\
\hline
\end{tabular}

$\mathrm{N}$, node.

Table 5 Definition of distant metastasis

\begin{tabular}{ll}
\hline M category & M description \\
\hline M0 & No pleural, pericardial, or distant metastasis \\
M1 & Pleural, pericardial, or distant metastasis \\
M1a & Separate pleural or pericardial nodule(s) \\
M1b & $\begin{array}{l}\text { Pulmonary intraparenchymal nodule or distant } \\
\text { organ metastasis }\end{array}$ \\
\hline
\end{tabular}

$\mathrm{M}$, metastasis.

\section{Nonepithelial tumors}

\section{Lymphoma}

Lymphoma may involve the thymus as part of a disseminated disease or as an isolated site. Hodgkin disease represents the majority of thymic lymphomas, with nodular sclerosis being the most common histologic finding identified in the thymus, while non-Hodgkin lymphoma represents $15-25 \%$ and the two most common forms include diffuse large B-cell
Table 6 Thymic stage groups

\begin{tabular}{lccc}
\hline T & N & M & Stage group \\
\hline T1a,b & N0 & M0 & I \\
T2 & No & M0 & II \\
T3 & N0 & M0 & IIIA \\
T4 & No & M0 & IIIB \\
Any T & N1 & M0 & IVA \\
Any T & N0,1 & M1a & IVA \\
Any T & N2 & M0, M1a & IVB \\
Any T & Any N & M1b & IVB \\
\hline
\end{tabular}

lymphoma and T-cell lymphoblastic lymphoma (23).

\section{Germ cell tumors (GCTs)}

GCTs mainly grow in gonads and in the midline of the body as well and the mediastinum being the most common extragonadal site; in particular, they account for $10-15 \%$ of adult anterior mediastinal tumors in adults and $25 \%$ in children (2). Only $3 \%$ of them arise in the posterior mediastinum. Pathological classifications include teratomas and non-teratomatous GCTs (embryonal cell carcinoma, yolk sac tumor, choriocarcinoma and mixed types). Teratoma is the most common histologic type, counting about $70 \%$ of all GCTs; they are classified into three subtypes: mature, immature and those with additional malignant components (if it contains fetal or neuroendocrine tissue) and are usually benign but still 
Table 7 Imagine features of benign and malignant thymic lesions

\begin{tabular}{|c|c|c|}
\hline Technique & Benign imaging features & Malignant imaging features \\
\hline \multirow{2}{*}{ X-ray } & \multirow[t]{2}{*}{ It is almost impossible to distinguish between benign and malignant lesions } & Pleural nodes \\
\hline & & Elevation of the hemidiaphragm \\
\hline \multirow[t]{5}{*}{ CT } & Regular outline & Irregular outline \\
\hline & \multirow[t]{4}{*}{ Absent or moderate contrast enhancement } & Local invasion \\
\hline & & Contrast enhancement \\
\hline & & Increased IC on DECT \\
\hline & & Low PS on perfusion \\
\hline \multirow{2}{*}{ MRI } & \multirow[t]{2}{*}{ Regular outline } & Hypointensity in ADC map \\
\hline & & Necrosis and contrast enhancement \\
\hline PET & Low SUV & High SUV \\
\hline
\end{tabular}

CT, computed tomography; IC, iodine concentration; DECT, Dual energy CT; PS, permeability surface; MRI, magnetic resonance imaging; DWI, diffusion weighted imaging; ADC, apparent diffusion coefficient; PET, positron emission tomography; SUV, standardized uptake value.

they could have a low malignancy potential (3). The nonteratomatous GCTs are rare and malignant tumours which usually occur in young males and most frequently affect the anterosuperior mediastinum. They grow rapidly and develop bulky masses. Invasion of adjacent structures and distant metastasis may occur, such as pleural and pericardial effusions. Measuring alpha-fetoprotein (AFP) and human chorionic gonadotropin (b-hCG) levels is important when making the diagnosis.

Other differential diagnosis in anterior mediastinal masses could be lipoma, liposarcoma, lymphangioma, ectopic thyroid and parathyroid lesions and sarcoidosis (24-27).

\section{Imaging}

Imaging is pivotal in trying to characterize a mass of the anterior mediastinum. In Table 7 the main imaging features of benign and malignant thymic lesions are summarized considering the different imaging modalities.

\section{Chest X-ray}

Conventional radiology is the first imaging technique to perform in the suspicion of an anterior mediastinal mass; in fact, a large anterior mediastinal mass is readily identified by chest radiography as it typically appears as an extra soft tissue mass or opacity. Despite the density of a soft tissue mass is similar to the anterior mediastinal structures, the use of silhouette sign, which describes the loss of normal anatomical borders, increases the sensitivity of detecting abnormalities. For this reason, since the mass displaces the air-containing lung from the mediastinum, the normal border is lost. On the other hand, the identification of a small mediastinal mass requires more attention; in particular the presence of the anterior junction line, representing the point of contact between the anterior lungs and their pleural surfaces anterior to the cardiovascular structures, can help to exclude the presence of an anterior mediastinal mass; however, this line is seen only in $20 \%$ of normal chest radiographs (28). Occasionally advanced diseases may be detected on chest radiograph, manifesting as an irregular border with the lung, an elevation of the ipsilateral hemidiaphragm or pleural nodes, signifying direct lung invasion, phrenic nerve involvement and metastases, respectively (29). Subsequently it is essential to differentiate between thymic disease (benign or malignant) and non-thymic disease by cross-sectional imaging, CT or MRI (29). 

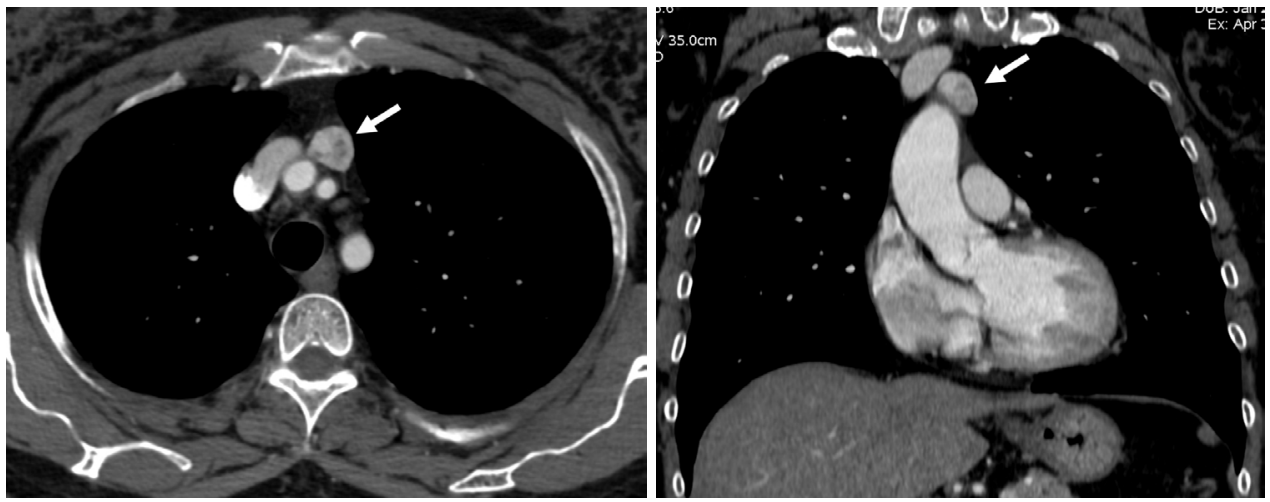

Figure 2 Contrast-enhanced CT showing a hypervascular nodule (arrows) of the anterior mediastinum with hypodense areas and smooth in its boundaries. The medical report suggests the diagnosis of thymoma; on the other hand, pathological evaluation after surgery reveals an ectopic thyroid. CT, computed tomography.

\section{CT, dual energy $C T$ and perfusion $C T$}

Although CT is typically considered the imaging modality to choose for the characterization of an anterior mediastinal mass and for the staging of the disease, the role of MRI imaging continues to expand (30). CT, thanks to its high spatial resolution, demonstrates not only the extent of the lesion and its relationship to adjacent structures (31) but also allows the visualization of some features of various mediastinal tumors to avoid an unnecessary biopsy or a surgical treatment. In particular, CT could help in differential diagnosis of anterior mediastinal masses on the basis of lesion composition, recognizing: (I) masses with fat attenuation: lipoma, liposarcoma, omental hernia, thymolipoma, teratoma; (II) masses with water attenuation: thymic cyst, lymphangioma, abscess; (III) masses with soft-tissue attenuation: thymic hyperplasia, thymic neoplasm, thyroid goiter, ectopic parathyroid adenoma, germ cells tumors, lymphoma; (IV) masses with calcific attenuation: treated malignancy, goiter, lymph nodes in granulomatosis infection or sarcoidosis-silicosis (2,3) (Figure 2). Totanarungroj et al. in 2010 evaluated 50 patients with an anterior mediastinal mass (thymoma, GCTs and lymphoma) who undergone chest CT scan trying to find CT features that may help in suggesting specific diagnosis between these tumors. The authors evaluated the presence of fat, cystic or calcific attenuation within the lesion or the presence of lymphadenopathy associated with the mass. Although the association of calcifications, fat and cyst attenuation is frequent in mature teratoma, there are some cases where these features lack; the combination of cystic component and calcification may be present in both GCTs and thymoma; calcifications are present both in benign and malignant thymoma; associated pathologic lymph nodes are frequent in lymphoma. The authors concluded that the only features that may help in giving a specific diagnosis of anterior mediastinal tumors are fat attenuation, which is highly suggestive for GCT, and associated mediastinal lymphadenopathy, which are indicative for mediastinal lymphomas (31). Hammer et al. identified 223 patients with anterior mediastinal mass and investigated several CT features for their association with histology. They found that lymphoma and GCTs tended to be larger than thymomas or benign lesions. Internal mammary, mediastinal and supraclavicular lymphadenopathy demonstrate a strong association with lymphoma; mediastinal encasement and lung invasion were also highly associated with lymphomas (32-34) (Figure 3).

The presence of interspersed fat inside the lesion is usually associated with a benign diagnosis (e.g., thymic hyperplasia). However, intralesional fat was also occasionally seen in malignant lesions such as lymphomas or thymomas, because of the invasion of mediastinal fat. Globular intralesional fat may be seen in certain GCTs (e.g., teratoma) (32) (Figure 4). Pleural metastases are frequent in thymomas and thymic carcinomas and usually manifest as nodular or lenticular lesions with or without pleural effusion, which should be differentiated from pleural plaques (35-37).

Thymoma, which represents $20 \%$ of anterior mediastinal neoplasms, typically appears on CT scan as a spherical or ovoidal, smooth, mediastinal mass, usually closely related to the superior pericardium, with post-contrast enhancement. It may show heterogeneous attenuation because of areas of hemorrhage and necrosis together with punctate, coarse 

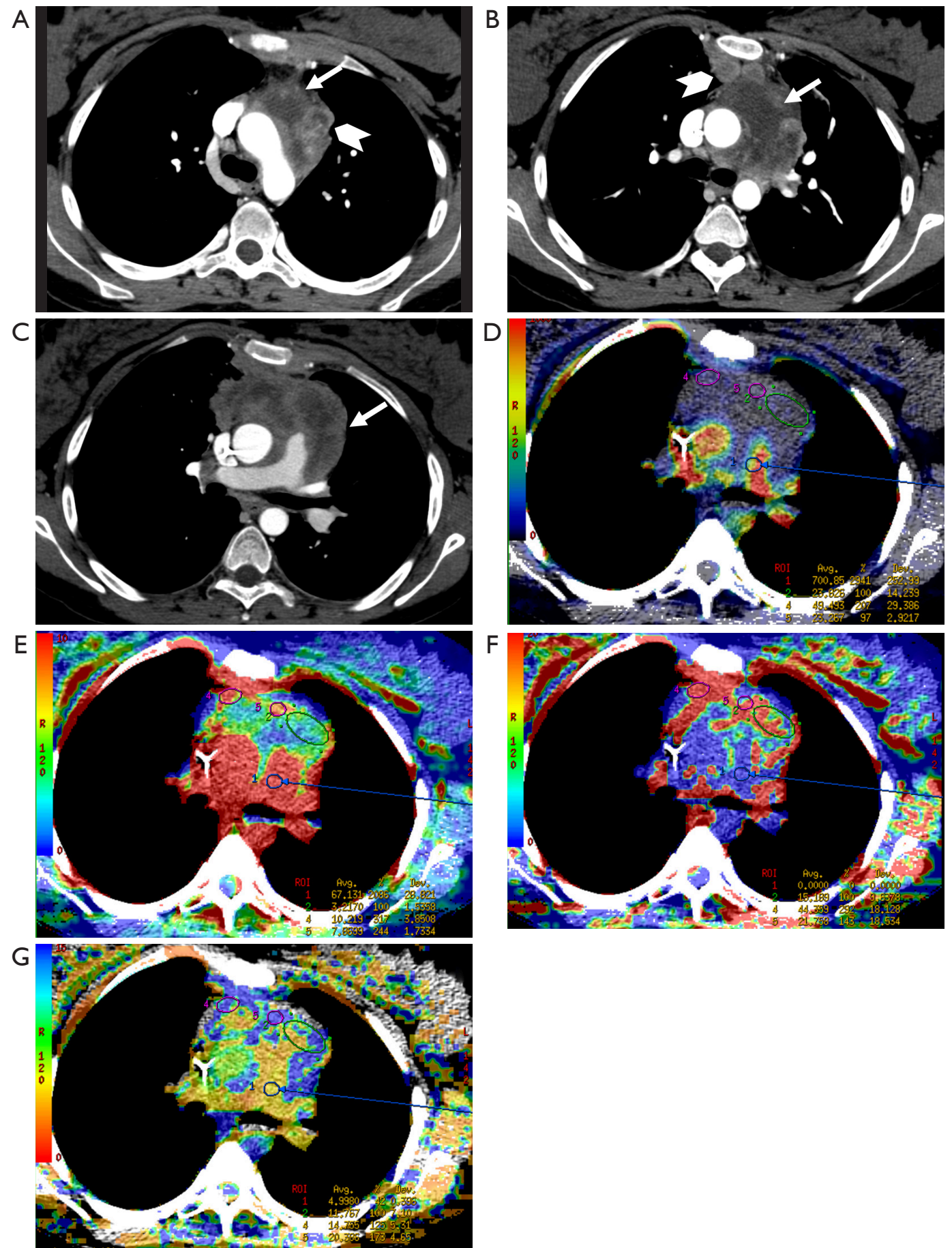

Figure 3 Morphological findings (A,B,C) and perfusion parameters [(D) blood flow; (E) blood volume; (F) permeability surface; (G) mean transit time] are consistent with diagnosis of lymphoma. Video-assisted thoracoscopic surgery revealed a primary mediastinal B-cell lymphoma. Contrast-enhanced CT scan accompanied by CT perfusion protocol show a heterogeneous neoplastic mass encasing the mediastinum (arrows in A,B,C) with lymphadenopaties (arrowheads in A and B). CT, computed tomography. 

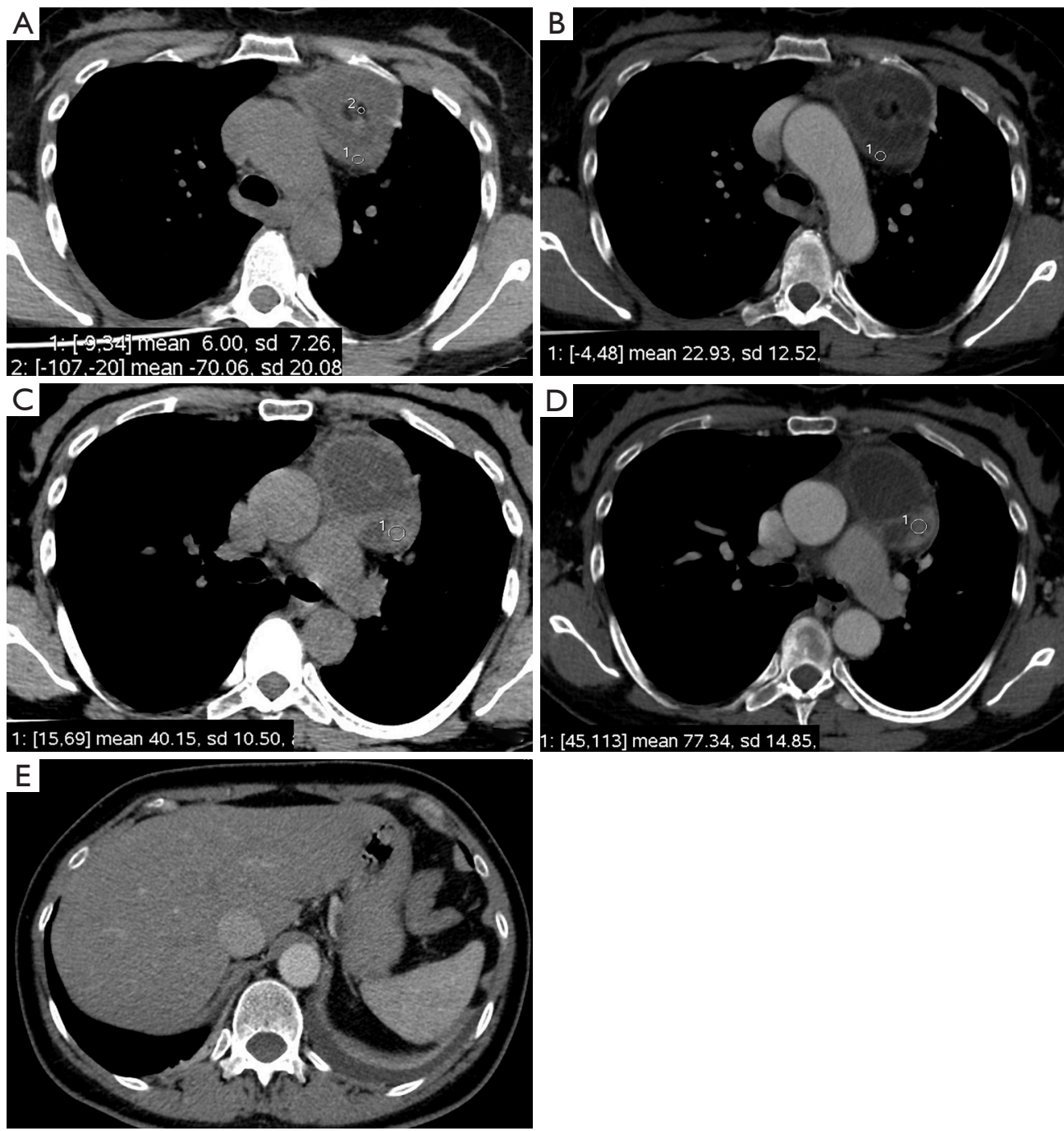

Figure 4 Chest pain and dyspnea in a 48-year-old woman. Chest CT images without (A,C) and with contrast (B,D) show a partially cystic lesion with thick wall in the anterior mediastinum. It is clear the contrast-enhancement of lesion wall and solid component (region of interest 1 in A,B,C,D). In the image without contrast it is clear the presence of macroscopic fat (region of interest 2 in A). There is a small amount of left pleural effusion (E). Surgery revealed a mature teratoma. CT, computed tomography.

or curvilinear calcifications $(29,38)$. Several studies have attempted to study the morphology of thymomas in order to improve preoperative staging accuracy (39-41). Priola $e t$ $a l$. and Tomiyama et al., Qu et al. evaluated retrospectively 50, 58 and 129 patients with thymoma respectively trying to distinguish stage I from advanced diseases. The authors found that obliteration of fat planes around the tumor was not helpful in distinguishing stage I from higher stages. However, they found that lobulated or irregular contours, cystic or necrotic regions within the tumor, together with multifocal calcifications were more suggestive of invasive neoplasia (40-42) (Figure 5). In another study, Marom et al., comparing the CT appearance of 99 thymomas, tried to differentiate early disease (stages I and II) from advanced disease, which requires neoadjuvant chemotherapy; the author found that only tumor diameter, infiltration of the fat surrounding the tumor and lobulated contours were associated with advanced disease (39).

More information about thymic neoplasm can be obtained with quantitative analysis of contrast medium uptake on dual energy computed tomography (DECT). In fact, this technique seems to be able to objectively 

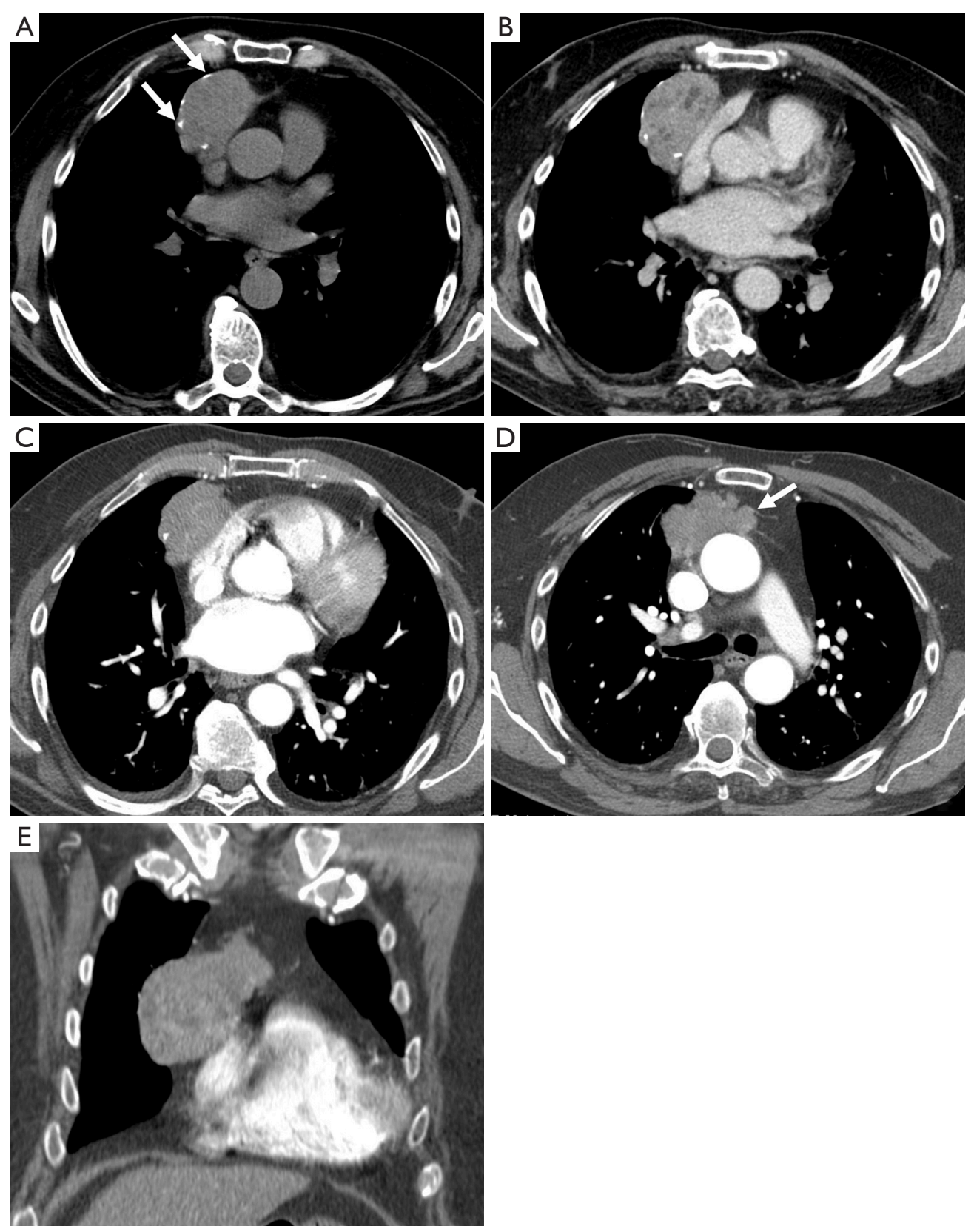

Figure 5 Neoplastic lesion of the anterior mediastinum located in the thymic lodge and adjacent to the pericardium. The lesion shows heterogeneous attenuation with coarse calcification (arrows in A) and irregular contours (arrow in D). Surgery revealed a thymoma (Masaoka stage IIB).

quantify iodine uptake of different lesions (43). Chang et al. investigated the role of DECT in the differentiation of thymic epithelial tumors and its capability to distinguish low from high risk tumors; they evaluated 37 thymic tumors with a qualitative and a quantitative analysis and they divided low risk thymomas (types $\mathrm{A}, \mathrm{AB}, \mathrm{B} 1$ ) from high risk thymomas (types B2, B3) and thymic carcinoma.

For the qualitative analysis, each lesion was characterized in term of size and volume; moreover, the radiologists evaluated internal characteristics of the tumor (contour, necrosis and calcification) and external characteristics (local invasion, metastasis, pericardial 
or pleural effusion and lymphadenopathies). There are significant differences in size between low risk thymomas and thymic carcinomas; moreover, tumor contour was significantly different in low risk thymomas and thymic carcinomas: all thymic carcinomas have irregular outline. On the other hand, no significant differences in term of size or contour were found between low risk thymomas and high risk thymoma and between high risk thymoma and thymic carcinoma (44).

The quantitative analysis was based on the evaluation of some parameters such as contrast Hounsfield unit (CHU), iodine concentration (IC) and iodine-related Hounsfield unit (IHU). On a dedicated workstation CHU was calculated by drawing a volume of interest (VOI) of the tumor on post-contrast CT images; IC is measured on the iodine map elaborated by a dedicated software of the workstation and IHU is calculated by the subtracting nonenhanced Hounsfield unit (HU) to the CHU.

The final analysis showed that there was a significant difference in IC and IHU between low risk thymoma and high risk thymoma and also between low risk thymoma and thymic carcinomas; however, there was no significant difference between high risk thymomas and thymic carcinomas and IC and IHU were lower in high risk thymomas and in thymic carcinomas than in low risk thymomas (44).

The reduction of IC and IHU was essentially due to the presence of abundant necrosis: this study suggests that these parameters allow a better definition on presence/absence of necrosis than just CHU and it concludes that DECT allows to evaluate both morphological characteristic and IC that can help to predict the WHO classification and the Masaoka-Koga stage.

These results were confirmed also by the study of Yan et al. that analyzed CHU, IC and IHU in post-contrast artery and venous phase CT; they confirmed that IHU and IC are higher in low risk than in high risk thymomas and thymic carcinomas both in artery and venous phase; however, the analysis of these parameters on venous phase seems to be more effective for the differential diagnosis (45).

CT perfusion, a functional imaging technique, providing quantitative data on tissue perfusion such as blood flow (BF), blood volume (BV) and permeability surface (PS), offers a different approach for preoperative diagnosis of mediastinal anterior masses (46). In fact, CT perfusion has been found useful in evaluating tumor angiogenesis, characterization, infiltration of tissue surrounding the lesion and tumor response to chemoradiotherapy (47-52). Bakan et al. recently investigated the possibility of this technique to differentiate thymoma from thymic hyperplasia, lymphoma, thymic carcinoma and invasive lung cancer involving the anterior mediastinum (Figure 6). The authors found a statistically significant difference in BV between thymoma and lymphoma (Figures 7,8). Moreover, comparison of perfusion values of thymoma with those of other malignant tumors revealed significantly higher $\mathrm{BF}$ and $\mathrm{BV}$ values in thymoma (46).

Texture analysis is an innovative technique that analyses the distribution and relationship of pixels in the images, providing data on tumor characteristics; in this sense, Iannarelli et al. analysed 16 patients affected by thymic tumors and they found a significant relationship between radiomics features and tumor necrosis, Masaoka classification and myasthenic syndrome (53); however, these results should be validated in a large case series and the technique is affected by low reproducibility among the different softwares employed $(54,55)$.

\section{MRI}

MR is another imaging modality that could be effective in distinguishing thymic epithelial neoplasm and other malignant tumors from benign lesions in the anterior mediastinum and can be used to characterize and stage thymic tumors in those patients with contraindications for contrast-enhanced CT, such as severe contrast allergy and/ or renal failure. MRI does not use ionizing radiations and can be safely used in young patients; however, since MR scan time is much longer than CT, an effective cooperation of the patient is pivotal for the success of the examination. Comparing to CT, MR is crucial for distinguishing normal thymus and thymic hyperplasia from small thymomas because of the high sensitivity of T1-weighted gradientecho images for detecting microscopic fat; since the normal thymus and thymic hyperplasia contain a small amount of fat, on T1-weighted out-phase images it is clearly visible a decreased signal intensity comparing to T1-weighted inphase images, whereas this finding lacks in case of thymic tumors (58) (Figures 9,10).

In this regard, a quantitative analysis of MR images is also possible by measuring the chemical shift ratio (CSR); the CSR is determined by comparing the signal intensity of the thymus gland with that of the paraspinal musculature on both T1-weighted in-phase and opposed-phase images (15). It is not routinely performed because there is no consensus nor accepted guidelines for measurements and 

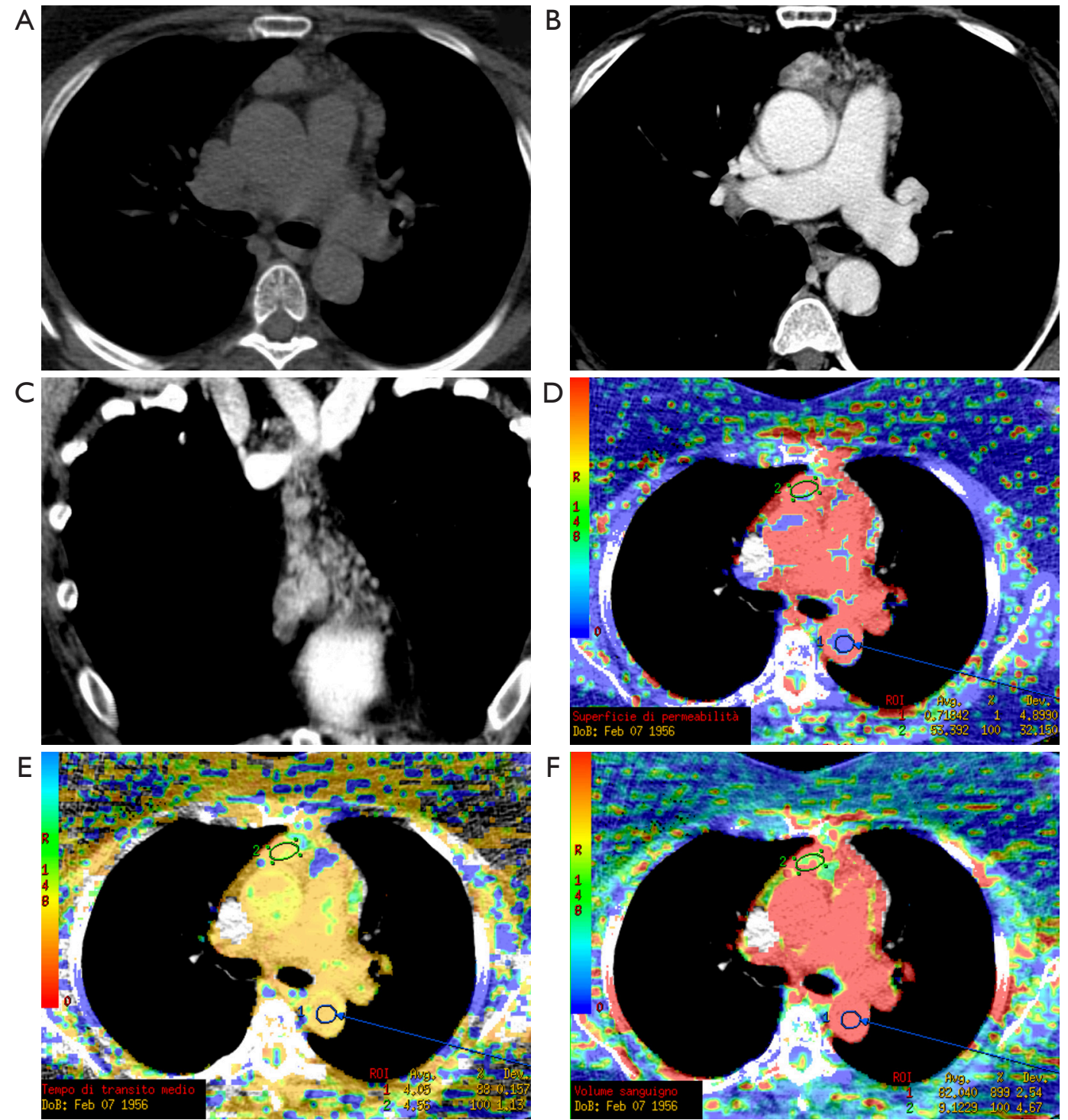

Figure 6 Occasional identification of multiple solid hypervascular nodules in the thymic lodge in an asymptomatic 53-year-old woman. CTperfusion parameters [(D) permeability surface; (E) mean transit time; (F) blood flow] do not allow a conclusive diagnosis. Surgery revealed a lymphoid thymic hyperplasia with florid germinal centers, consistent with autoimmune thymitis. CT, computed tomography.

interpretation of data but it has been demonstrated to improve the characterization of thymus abnormalities and in particular the differentiation between normal thymus or hyperplastic thymus from thymic neoplasms $(14,56)$. Thymoma on MR usually shows low to intermediate signal intensity on T1-weighted images and high signal intensity on T2-weighted images; however, signal intensity can be heterogeneous in tumors with necrosis, hemorrhage or cystic changes (29).
As on CT, some attempts have been made on MR for thymoma preoperative staging. Abdel Razek et al. tried to categorize 30 different thymic epithelial neoplasms using qualitative and quantitative analysis on MR imaging. On qualitative analysis, they demonstrated that lobulated margins are more often seen in low risk thymoma (types A, $\mathrm{AB}$ and $\mathrm{B} 1$ ) than in high risk thymoma (types $\mathrm{B} 2$ and $\mathrm{B} 3$ ) or thymic carcinoma, necrosis was present in all thymic carcinomas and more often in high risk thymoma than in 

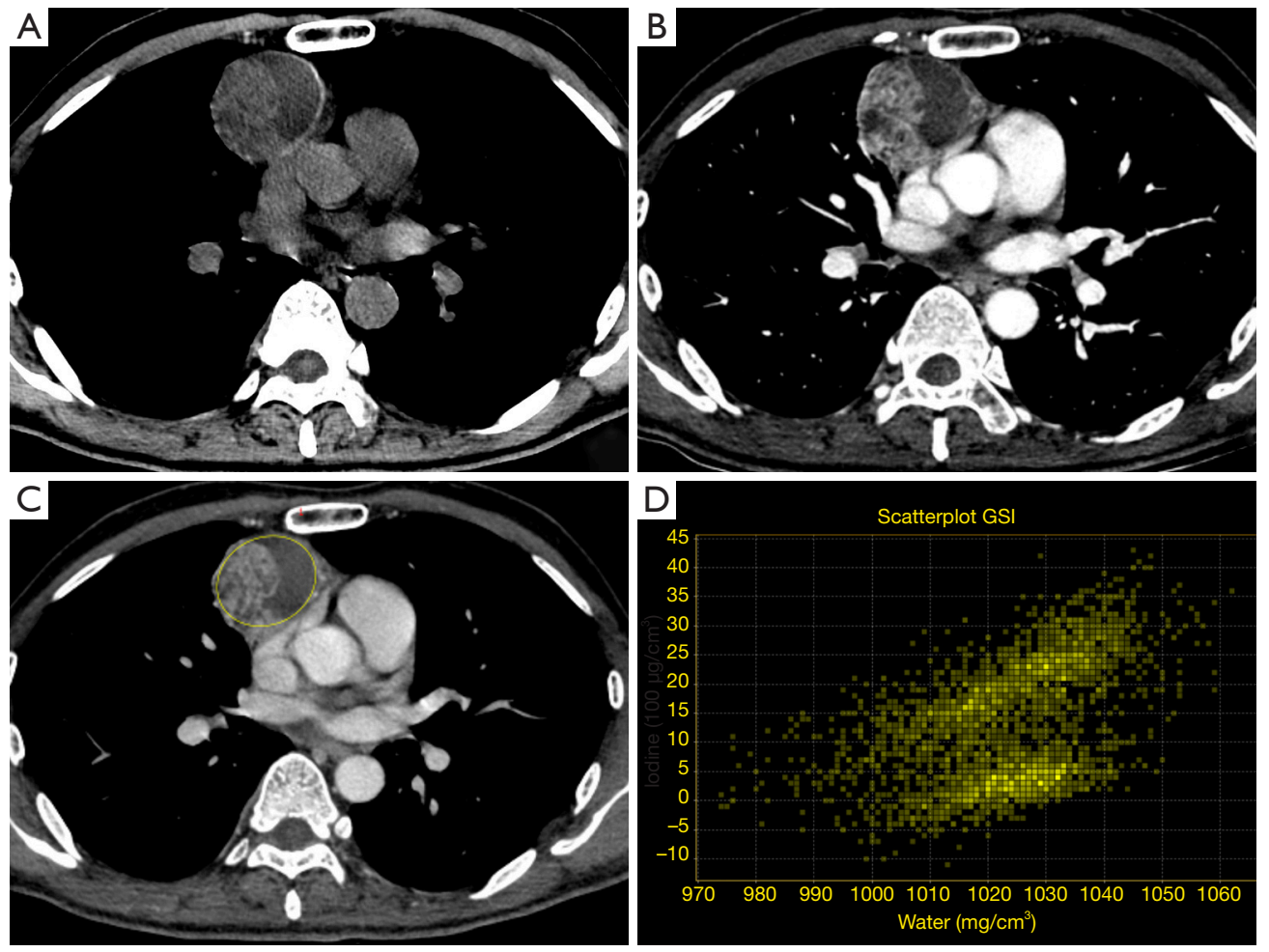

Figure 7 DECT in a 47-year-old man myasthenia gravis and polymyositis show a neoplastic lesion with a mixed component (solid and cystic) adjacent to the pericardium. The region of interest 1 in $\mathrm{C}$ and corresponding scatterplot (D) show shows the post-contrast iodine uptake within the lesion. DECT, dual energy computed tomography.

low risk thymoma, as well as pleural effusion (57).

The quantitative analysis was performed using diffusion weighted images (DWI). The image quality and diagnostic capability of MR have improved and the use of DWI in diagnosis of neoplasms is largely diffuse; recently it has been introduced in thoracic MR study.

In their study Abdel Razek et al. demonstrated that there was a significant statistical difference of the apparent diffusion coefficient (ADC) values between high risk and low risk thymomas; in particular ADC values were higher in low risk thymomas $\left(1.30 \times 10^{-3} \mathrm{~mm}^{2} / \mathrm{s}\right.$ $\pm 0.08)$ than in high risk thymomas $\left(1.16 \times 10^{-3} \mathrm{~mm}^{2} / \mathrm{s}\right.$ $\pm 0.07)$ and thymic carcinomas $\left(1.18 \times 10^{-3} \mathrm{~mm}^{2} / \mathrm{s}\right.$ \pm 0.23 ) (57). They also evaluated the possibility to distinguish Masaoka-Koga stage classes by using ADC values; I and II stages showed higher ADC values than those of III and IV stages: $1.31 \times 10^{-3} \mathrm{~mm}^{2} / \mathrm{s} \pm 0.08$ for early stages (I and II), $1.18 \times 10^{-3} \mathrm{~mm}^{2} / \mathrm{s} \pm 0.13$ for advanced stages (III and IV) (57). The authors concluded that the best cut-off for ADC value used to differentiate low risk thymoma from high risk thymoma and thymic carcinomas was $1.22 \times 10^{-3} \mathrm{~mm}^{2} / \mathrm{s}$ (accuracy $88 \%$ and $86 \%$; sensitivity $75 \%$ and $87 \%$; specificity $85 \%$ and $88 \%$ ) (57). Another advantage of DWI is that, conversely to chemical shift MRI, it seems to be able to differentiate normal thymus from thymic hyperplasia by using ADC values which are higher in normal thymus $\left(2.10 \pm 0.23 \times 10^{-3} \mathrm{~mm}^{2} / \mathrm{s}\right.$ and $1.86 \pm 0.17 \times 10^{-3} \mathrm{~mm}^{2} / \mathrm{s}$ ) (14). Post-contrast dynamic MR imaging is also useful for the differential diagnosis of anterior mediastinal tumors: in this regard Reubi et al. demonstrated that mean time to peak (MTP) of the time intensity curve was different between thymomas $(1.5 \mathrm{~min})$ and non-thymoma tumors. They also demonstrated that thymomas stage I/II have a MTP significantly shorter $(1.3 \mathrm{~min})$ than stage III thymomas $(2.5 \mathrm{~min})(58)$. Moreover, particular MR sequences used to study lymphatic system (magnetic resonance lymphangiography) that adopt an effective echo time close to lymphatic T2, may be performed in the suspicion of lymphangioma $(25,59)$. Concerning the comparison between MR and CT for diagnosing anterior mediastinal masses, 

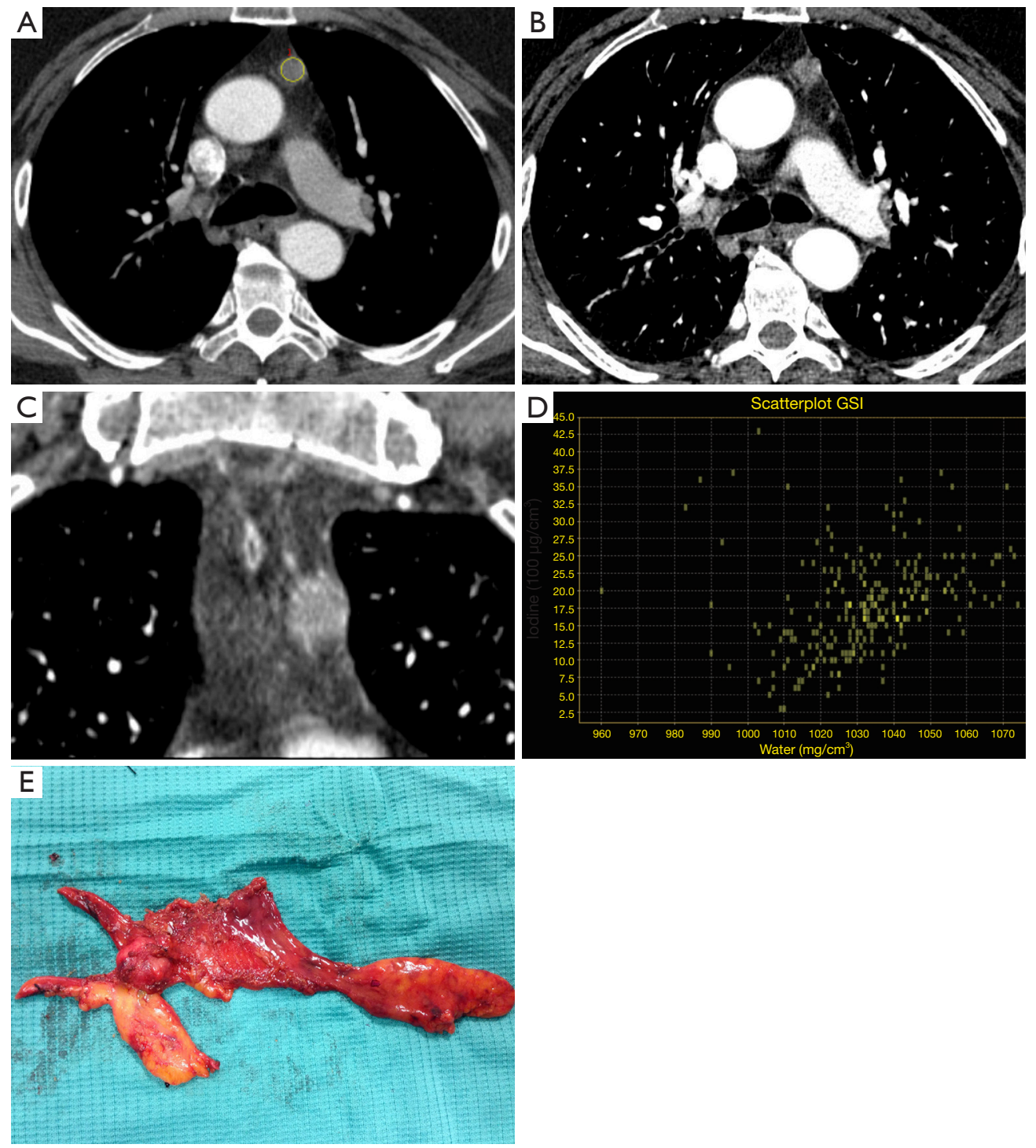

Figure 8 Dual-energy post-contrast axial [(A) $77 \mathrm{keV}$; (B) $40 \mathrm{keV}$ ] and coronal [(C) $40 \mathrm{keV}$ ] chest CT show a solid lesion with finely irregular borders in the thymic lodge in a 67-year-old-man. The scatterplot (D) show a slight iodine uptake within the lesion; (E) pathological analysis after surgery reveals a thymic basaloid carcinoma (Masaoka stage I). CT, computed tomography.

Seki et al. compared DWI-MR imaging with multidetector row CT; they demonstrated that ADC values of the anterior mediastinal solitary tumors were equally effective, compared with CT for distinguishing thymic neoplasms from other anterior mediastinal tumors and for differentiating thymic WHO categories and clinical staging (early $v s$. advanced); therefore they asserted that DWI can be performed to complete a CT scan when the CT characteristics of a lesion do not permit a conclusive diagnosis (17).

\section{Positron emission tomography (PET)}

Little is known about the diagnostic performance of 18-fluorodeoxyglucose (FDG) PET-CT in the anterior mediastinal tumors. It is well known that 18-FDG PET-CT is strongly recommended in diagnosis, early assessment/ restaging and follow up of Hodgkin lymphoma and avid non-Hodgkin lymphoma (60). Because one of the possible differential diagnosis for an anterior mediastinal mass is lymphoma, especially in young people, (approximately $20 \%$ of mediastinal neoplasms in adult and $50 \%$ of 

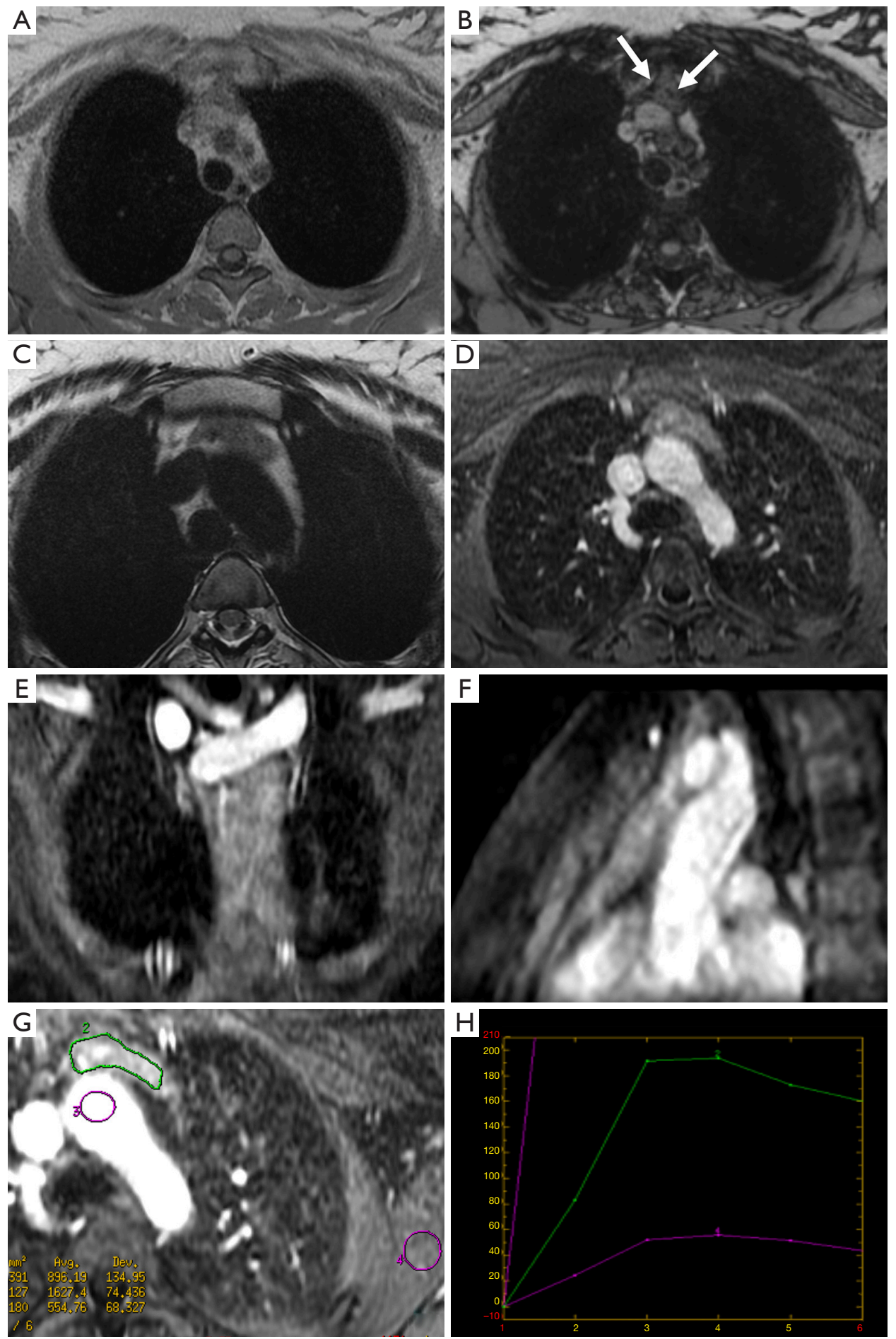

Figure 9 Chest MR in a 27-year-old woman with myasthenia gravis. Axial T1-gradient in-phase (A) and out-phase (B) and T2-weight (C) images show a substantially symmetrical enlargement of the thymus gland; comparing T1 in-phase and out-phase images, small arear with signal drop (arrows in B) are evident in the latter sequences because of the presence of microscopic fat. Post-contrast T1 axial (D), coronal oblique (E) and sagittal (F) images show a homogeneous enhancement of the thymus gland. Curves derived from dynamic sequences show a progressive enhancement of thymic tissue followed by a mild wash-out (region of interest 2 in $\mathrm{G}$ and green line in $\mathrm{H}$ ). Region of interest 4 with the corresponding violet line in $\mathrm{H}$ show the minor enhancement of subscapularis muscle for comparison. Pathological evaluation after surgery revealed a lymphoid hyperplasia. MR, magnetic resonance. 

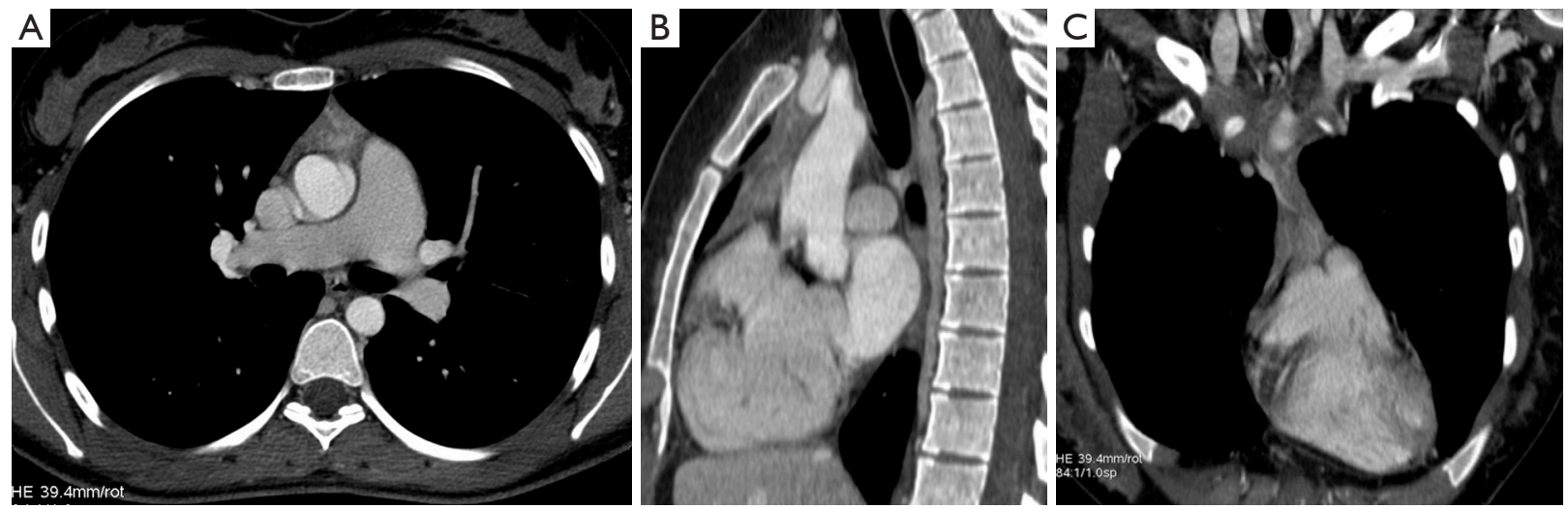

Figure 10 Contrast-enhanced CT of the chest in a 29-year-old woman with myasthenia gravis. Axial (A), sagittal (B) and coronal (C) images show a small amount of tissue with triangular shape in the thymic lodge, consistent with thymic hyperplasia. Pathological evaluation after surgery revealed a thymic hyperplasia with expansion of the medullary areas.

neoplasms in children) (57) once an anterior mediastinal mass is incidentally seen on a chest X-ray or CT some diagnostic workup includes PET-CT. Proli et al. conduced a retrospective study on a cohort of 134 patients who underwent surgery for an anterior mediastinal mass and received 18-FDG PET-CT as a part of their workup. PETCT was positive in 110 and negative in 24 of 134 patients and the final histological analysis revealed 101 malignant conditions (thymoma, thymic carcinoma, lymphoma) and 33 non-malignant conditions (thymic hyperplasia and thymic cyst); the statistical analysis demonstrated a sensitivity of $83 \%$, a specificity of $58 \%$, a positive and negative predictive value of $90 \%$ and $42 \%$ respectively (61). Therefore, this imaging modality does not have a role in routine clinical practice in order to assess the malignant potential of an anterior mediastinal mass.

\section{The role of imaging guided biopsy}

The effort to achieve a diagnosis in case of anterior mediastinal lesions have to be balanced to the risk of tumor cells seeding in the chest wall. Above all, thymomas are associated with a high risk of local recurrence and for this reason capsulated lesions, expected to be thymoma stage Masaoka III, are preferred to be treated with upfront surgery avoiding the risk of seeding due to a diagnostic invasive procedure (62). The invasive preoperative diagnosis is generally reserved to patients affected by invasive mediastinal lesions and in those with a high suspect of lymphoproliferative disease (63). For the first ones, the diagnosis is mandatory for a preoperative induction treatment before surgery or a definitive chemoradiotherapy (64); for the second ones, is mandatory to address the chemotherapy regimen. In all cases, a cytological evaluation is considered inappropriate and a histologic sample is mandatory just only to discriminate between a thymic carcinoma and a lymphoproliferative disease (65). The anterior position of these lesions makes the surgical biopsy easy with a good patient compliance, however, when feasible, a CT or echo guided needle biopsy seems reach the same accuracy of an open biopsy avoiding patient hospitalization and general anesthesia (63).

\section{Conclusions}

Awareness of the embryologic, histologic, and normal morphologic features of the thymus is essential for recognizing the normal thymus and suspecting the different thymic diseases. Furthermore, since imaging could help in differential diagnosis of thymic diseases, staging, prognostication as well as in the followup monitoring for recurrence of malignant thymic diseases, radiologists should be aware about the role and possibilities of different diagnostic techniques. Moreover, because treatment of thymic pathologies could be different (i.e., patients with advanced thymoma receive neoadjuvant chemotherapy or lymphoma that could avoid surgery), radiologists should be familiar with the staging system of thymoma in order to identify such patients before surgery and as much as possible should be familiar with the imaging features of different thymic pathologies, in order to avoid unnecessary surgery. 


\section{Acknowledgments}

None.

\section{Footnote}

Conflicts of Interest: The authors have no conflicts of interest to declare.

\section{References}

1. Nishino M, Ashiku SK, Kocher ON, et al. The Thymus: A Comprehensive Review-Erratum. Radiographics 2017;37:1004.

2. Juanpere $\mathrm{S}$, Cañete $\mathrm{N}$, Ortuño $\mathrm{P}$, et al. A diagnostic approach to the mediastinal masses. Insights Imaging 2013;4:29-52.

3. Shahrzad M, Le TS, Silva M, et al. Anterior Mediastinal Masses. AJR Am J Roentgenol 2014;203:W128-38.

4. Felson B. The mediastinum. Semin Roentgenol 1969;4:41-58.

5. Whitten CR, Khan S, Munneke GJ, et al. A diagnostic approach to mediastinal abnormalities. Radiographics 2007;27:657-71.

6. Fujimoto K, Hara M, Tomiyama N, et al. Proposal for a new mediastinal compartment classification of transverse plane images according to the Japanese Association for Research on the Thymus (JART) General Rules for the Study of Mediastinal Tumors. Oncol Rep 2014;31:565-72.

7. Carter BW, Tomiyama N, Bhora FY, et al. A modern definition of mediastinal compartments. J Thorac Oncol 2014;9:S97-101.

8. Carter BW, Benveniste MF, Madan R, et al. ITMIG Classification of Mediastinal Compartments and Multidisciplinary Approach to Mediastinal Masses. Radiographics 2017;37:413-36.

9. Van Schil PE, Heyman S. Invited Commentary on "ITMIG Classification of Mediastinal Compartments and Multidisciplinary Approach to Mediastinal Masses". Radiographics 2017;37:436-8.

10. Rusch VW, Asamura H, Watanabe $\mathrm{H}$, et al. The IASLC lung cancer staging project. A proposal for a new international lymph node map in the forthcoming seventh edition of the TNM classification for lung cancer. J Thorac Oncol 2009;4:568-77.

11. Volterrani L, Mazzei MA, Banchi B, et al. MSCT multicriteria: a novel approach in assessment of mediastinal lymph node metastases in non-small cell lung cancer. Eur J
Radiol 2011;79:459-66.

12. Tini $P$, Nardone $V$, Pastina $P$, et al. The effects of radiotherapy on the survival of patients with unresectable non-small cell lung cancer. Expert Rev Anticancer Ther 2018;18:593-602.

13. Fraser RG, Paré JAP. The normal chest. Diagnosis of Diseases of the Chest. 2nd edition. Philadelphia: WB Saunders Co.; 1978:813.

14. Priola AM, Gned D, Veltri A, et al. Chemical shift and diffusion-weighted magnetic resonance imaging of the anterior mediastinum in oncology: Current clinical applications in qualitative and quantitative assessment. Crit Rev Oncol Hematol 2016;98:335-57.

15. Priola AM, Priola SM, Gned D, et al. Nonsuppressing normal thymus on chemical-shift MR imaging and anterior mediastinal lymphoma: differentiation with diffusionweighted MR imaging by using the apparent diffusion coefficient. Eur Radiol 2018;28:1427-37.

16. Engels EA. Epidemiology of thymoma and associated malignancies. J Thorac Oncol 2010;5:S260-5.

17. Seki S, Koyama H, Ohno Y, et al. Diffusion-weighted MR imaging vs. Multi-detector row CT:Direct comparison of capability for assessment of management needs for anterior mediastinal solitary tumors. Eur J Radiol 2014;83:835-42.

18. Regnard JF, Magdeleinat P, Dromer C, et al. Prognostic factors and long-term results after thymoma resection:a series of 307 patients. J Thorac Cardiovasc Surg 1996;112:376-84.

19. Marx A, Ströbel P, Badve SS, et al. ITMIG consensus statement on the use of the WHO histological classification of thymoma and thymic carcinoma:refined definitions, histological criteria, and reporting. J Thorac Oncol 2014;9:596-611.

20. Jung KJ, Lee KS, Han J, et al. editors. Malignant thymic epithelial tumors: CT-pathologic correlation. AJR Am J Roentgenol 2001;176:433-9.

21. Amin MB, Edge SB, Greene FL, et al. AJCC Cancer Staging Manual. 8th edition. New York: Springer, 2017.

22. Ried M, Eicher MM, Neu R, et al. Evaluation of the new TNM-staging system for thymic malignancies:impact on indication and survival. World J Surg Oncol 2017;15:214.

23. Boger-Megiddo I, Apter S, Spencer JA, et al. Is chest CT sufficient for follow-up of primary mediastinal B-cell lymphoma in remission? AJR Am J Roentgenol 2002;178:165-7.

24. Mazzei MA, Gentili F, Mazzei FG, et al. High-resolution MR lymphangiography for planning lymphaticovenous anastomosis treatment: a single-centre experience. Radiol 
Med 2017;122:918-27.

25. Mazzei FG, Gentili F, Guerrini S, et al. MR

Lymphangiography: A Practical Guide to Perform It and a Brief Review of the Literature from a Technical Point of View. Biomed Res Int 2017;2017:2598358.

26. Gennaro P, Borghini A, Chisci G, et al. Could MRI visualize the invisible? An Italian single center study comparing magnetic resonance lymphography (MRL), super microsurgery and histology in the identification of lymphatic vessels. Eur Rev Med Pharmacol Sci 2017;21:687-94.

27. Mazzei MA, Gentili F, Tini P, et al. Multimodality imaging assessment of Malignant Pleural Mesothelioma. In: Giordano A, Franco R. editors. Malignant Pleural Mesothelioma: A Guide for Clinicians. Academic Press, 2019:19-34.

28. Carter BW, Okumura M, Detterbeck FC, et al. Approaching the patient with an anterior mediastinal mass:a guide for radiologists. J Thorac Oncol 2014;9:S110-8.

29. Marom EM. Advances in thymoma imaging. J Thorac Imaging 2013;28:69-80; quiz 81-3.

30. Carter BW, Lichtenberger JP 3rd, Benveniste MF. MR Imaging of Thymic, Epithelial Neoplasms. Top Magn Reson Imaging 2018;27:65-71.

31. Totanarungroj K, Watcharaporn C, Muangman N. Helpful CT findings for giving specific diagnosis of anterior mediastinal tumors. J Med Assoc Thai 2010;93:489-96.

32. Hammer MM, Miskin N, Madan R, et al. Predictive Features for Anterior Mediastinal Mass Diagnoses. J Comput Assist Tomogr 2019;43:98-103.

33. Volterrani L, Mazzei MA, Giordano N, et al. Magnetic resonance imaging in Tietze's syndrome. Clin Exp Rheumatol 2008;26:848-53.

34. Mazzei MA, Volterrani L. Errors in multidetector row computed tomography. Radiol Med 2015;120:785-94.

35. Mazzei MA, Contorni F, Gentili F, et al. Incidental and Underreported Pleural Plaques at Chest CT:Do Not Miss Them-Asbestos Exposure Still Exists. Biomed Res Int 2017;2017:6797826.

36. Bennett D, Fossi A, Refini RM, et al. Posttransplant solid organ malignancies in lung transplant recipients:a singlecenter experience and review of the literature. Tumori 2016;102:574-81.

37. Mazzei MA, Sartorelli P, Bagnacci G, et al. Occupational Lung Diseases: Underreported Diagnosis in Radiological Practice. Semin Ultrasound CT MR 2019;40:36-50.

38. Mignarri A, Gentili F, Masia F, et al. Imaging of the thymus in myotonic dystrophy type 1 . Neurol Sci 2018;39:347-51.

39. Marom EM, Milito MA, Moran CA, et al. Computed tomography findings predicting invasiveness of thymoma. J Thorac Oncol 2011;6:1274-81.

40. Priola AM, Priola SM, Di Franco M, et al. Computed tomography and thymoma: distinctive findings in invasive and noninvasive thymoma and predictive features of recurrence. Radiol Med 2010;115:1-21.

41. Tomiyama N, Muller NL, Ellis SJ, et al. Invasive and noninvasive thymoma: distinctive CT features. J Comput Assist Tomogr 2001;25:388-93.

42. Qu YJ, Liu GB, Shi HS, et al. Preoperative CT findings of thymoma are correlated with postoperative masaoka clinical stage. Acad Radiol 2013;20:66-72.

43. Mazzei MA, Gentili F, Volterrani L. Dual-Energy CT iodine mapping and 40-keV monoenergetic applications in the diagnosis of acute bowel ischemia:a necessary clarification. AJR Am J Roentgenol 2019;212:W93-4.

44. Chang S, Hur J, Im DJ, et al. Volume-based quantification using dual-energy computed tomography in the differentiation of thymic epithelial tumours:an initial experience. Eur Radiol 2017;27:1992-2001.

45. Yan WQ, Xin YK, Jing Y, et al. Iodine Quantification Using Dual-Energy Computed Tomography for Differentiating Thymic Tumors. J Comput Assist Tomogr 2018;42:873-80.

46. Bakan S, Kandemirli SG, Dikici AS, et al. Evaluation of anterior mediastinal solid tumors by CT perfusion: a preliminary study. Diagn Interv Radiol 2017;23:10-4.

47. Mazzei MA, Preda L, Cianfoni A, et al. CT perfusion: technical developments and current and future applications. Biomed Res Int 2015;2015:397521.

48. Mazzei FG, Volterrani L, Guerrini S, et al. Reduced time CT perfusion acquisitions are sufficient to measure the permeability surface area product with a deconvolution method. Biomed Res Int 2014;2014:573268.

49. Mazzei FG, Mazzei MA, Cioffi Squitieri N, et al. CT perfusion in the characterisation of renal lesions:an added value to multiphasic CT. Biomed Res Int 2014;2014:135013.

50. Mazzei MA, Cioffi Squitieri N, Guerrini S, et al. Quantitative CT perfusion measurements in characterization of solitary pulmonary nodules: new insights and limitations. Recenti Prog Med 2013;104:430-7.

51. Mazzei MA, Squitieri NC, Sani E, et al. Differences in perfusion CT parameter values with commercial software 
upgrades: a preliminary report about algorithm consistency and stability. Acta Radiol 2013;54:805-11.

52. Volterrani L, Mazzei MA, Fedi M, et al. Computed tomography perfusion, using first pass methods for lung nodule characterization: limits and implications in radiologic practice. Invest Radiol 2009;44:124; author reply 124.

53. Iannarelli A, Sacconi B, Tomei F, et al. Analysis of CT features and quantitative texture analysis in patients with thymic tumors: correlation with grading and staging. Radiol Med 2018;123:345-50.

54. Mazzei MA, Bagnacci G, Gentili F, et al. Gastric Cancer Maximum Tumour Diameter Reduction Rate at CT Examination as a Radiological Index for Predicting Histopathological Regression after Neoadjuvant Treatment: A Multicentre GIRCG Study. Gastroenterol Res Pract 2018. doi: 10.1155/2018/1794524.

55. Mazzei MA, Nardone V, Di Giacomo L, et al. The role of delta radiomics in gastric cancer. Quant Imaging Med Surg 2018;8:719-21.

56. Inaoka $\mathrm{T}$, Takahashi $\mathrm{K}$, Mineta $\mathrm{M}$, et al. Thymic hyperplasia and thymus gland tumors: differentiation with chemical shift MR imaging. Radiology 2007;243:869-76.

57. Abdel Razek AAK, Khairy M, Nada N. Diffusion-weighted MR Imaging in Thymic Epithelial Tumors: Correlation with World Health Organization Classification and Clinical Staging. Radiology 2014;273:268-75.

58. Reubi JC, Waser B, Horisberger U, et al. In vitro

Cite this article as: Gentili F, Pelini V, Lucii G, Luzzi L, Mazzei FG, Fausto A, Volterrani L, Mazzei MA. Update in diagnostic imaging of the thymus and anterior mediastinal masses. Gland Surg 2019;8(Suppl 3):S188-S207. doi: 10.21037/ gs.2019.05.06 autoradio- graphic and in vivo scintigraphic localization of somatostatin receptors in human lymphatic tissue. Blood $1993 ; 82: 2143-51$.

59. Crescenzi R, Donahue PMC, Hartley KG, et al. Lymphedema evaluation using noninvasive $3 \mathrm{~T}$ MR lymphangiography. J Magn Reson Imaging 2017;46:1349-60.

60. Cheson BD, Fisher RI, Barrington SF, et al.

Recommendations for initial evaluation, staging, and response assessment of Hodgkin and non-Hodgkin lymphoma:t he Lugano classification. J Clin Oncol 2014;32:3059-68.

61. Proli C, De Sousa P, Jordan S, et al. A diagnostic cohort study on the accuracy of 18-fluorodeoxyglucose (18 FDG) positron emission tomography (PET)-CT for evaluation of malignancy in anterior mediastinal lesions: The DECiMaL study. BMJ Open 2018;8:e019471.

62. Detterbeck FC, Zeeshan A. Thymoma: current diagnosis and treatment. Chin Med J (Engl) 2013;126:2186-91.

63. Ried M, Marx A, Götz A, et al. State of the art:diagnostic tools and innovative therapies for treatment of advanced thymoma and thymic carcinoma. Eur J Cardiothorac Surg 2016;49:1545-52.

64. Ströbel P, Marx A, Zettl A, et al. Thymoma and thymic carcinoma: an update of the WHO Classification 2004. Surg Today 2005;35:805-11.

65. Müller-Hermelink HK, Marx A. Thymoma. Curr Opin Oncol 2000;12:426-33. 